\title{
Vote and Be Heard: Adding Back-Channel Signals to Social Mirrors
}

\author{
Tony Bergstrom and Karrie Karahalios \\ Department of Computer Science \\ University of Illinois at Urbana-Champaign \\ \{abergst2, kkarahal\}@cs.uiuc.edu
}

\begin{abstract}
In face-to-face group situations, social pressure and organizational hierarchy relegate the less outspoken to silence, often resulting in fewer voices, fewer ideas, and groupthink. However, in mediated interaction like email, more people join in the discussion to offer their opinion. With this work, we aim to combine the benefits of mediated communication with the benefits and affordances of face-to-face interaction by adding a mediated back-channel. We describe Conversation Votes, a tabletop system that augments verbal conversation with a shared anonymous back-channel to highlight agreement. We then discuss a study of our design with groups engaged in repeated discussion. Our results show that anonymous visual back-channels provide a medium for the underrepresented voices of a conversation and balances interaction among all participants.
\end{abstract}

Keywords: Anonymous, back-channel, collocated, debate, feedback, voting.

\section{Introduction}

This work introduces an anonymous visual back-channel as a cue to shape the understanding of conversation in groups. A cue is a non-verbal communication such as a gesture, a facial expression, body posture, movement, or tone of voice [1][2][3][4][5]. Visual cues can enhance words or make their own statement: the meaning of "I love to work" can be redefined by rolling one's eyes [4]. Similar cues allow us to successfully negotiate social interactions, save face [3], and to coordinate actions quickly and efficiently [6]. In some cases, such as communicating feelings, non-verbal and visual communications are significantly more reliable indicators than the spoken word alone [5].

Considerable work has investigated the creation and conveyance of new conversational cues in remote spaces [7][8][9][10]. However, less work investigates their use in collocated spaces. One goal for this work is to enable new back-channel cues that communicate as effectively as traditional back-channel cues [11] but at a lowered social cost via anonymity. When speaking or gesturing, an individual draws attention to himself and affects his social persona, for better or worse [2]. The weight of being judged and the stigma associated with a mistake regulates speakers to silence. As US President Abraham Lincoln said, "It is better to remain silent and be thought a fool than to speak out and remove all doubt." While a person's image could benefit by adhering to this advice, the net result encourages silence and reliance on the rest of the group [12]. 


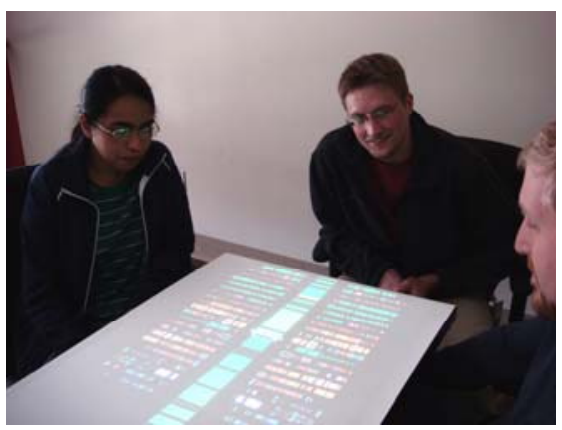

(a) Table in use

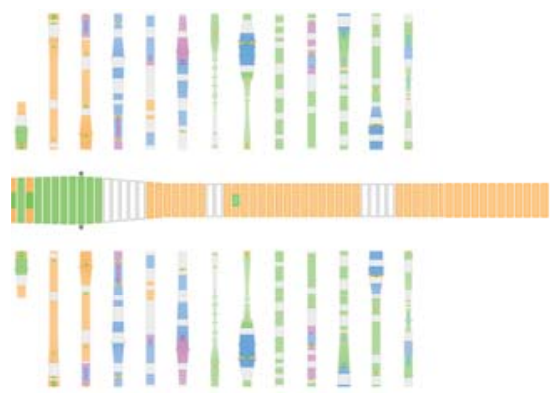

(b) Visualization

Fig. 1. Above we see a picture of the Conversation Votes table during a conversation. The most current interaction is highlighted by the larger horizontal central history. To provide a longer historical context, past minutes are mirrored in the historical stacks (more in Figures 2a, 2b).

The pressure to conform is less prevalent in anonymous and asynchronous interactions than in face-to-face or group interaction [13]. Employees in an organization withhold disagreements from employers in face-to-face meetings that they will divulge via email [10][14]. We designed Conversation Votes to allow similar, impersonal, feedback in a collocated environment (Figure 1a).

In the following sections, we will discuss previous work in face-to-face interaction, group awareness, and aural augmentation. We next describe the Conversation Votes visualization, focusing on design aspects for audio depiction and anonymous feedback in a face-to-face setting. This is followed by a study of the Conversation Votes that addresses questions of how participants utilize the visualization to understand their co-located peers. We conclude by discussing our results that show the underrepresented viewpoints become salient with a new anonymous 'visual voice.'

\section{Related Work}

Work related to Conversation Votes covers a wide range of areas. We briefly touch on three broad areas that are most relevant: Face to face interaction, group awareness, and aural augmentation.

\subsection{Face to Face}

With any social situation, appearance is important. A person changes behavior to fit the audience and make a good impression [2,3]. In the workplace, coworkers might be casual with each other, but only when it does not reflect negatively on the individual employees. If a distinguished guest is introduced, social protocol becomes more formal and distant.

A person will adapt their persona to be seen as amiable and intelligent in the eyes of the guest [15][16]. Professional vocabulary and mannerisms replace the normal signals; individuals say 'sir' or are overly attentive as a sign of respect [17]. However, 
communication is many faceted and not limited to spoken words. When listening to the guest, back-channels show interest and attention. These could be short phrases like 'yeah' or 'really?' but could also be a non-verbal smile or a head nod acting as a signal.

Evolutionary biology derived signaling theory to acknowledge both intended and unintended signals. When speaking to the guest, the intention may be to come across as interested and engaged, but unintended actions can betray a facade [18]. For example, checking the time might demonstrate the desire to leave or tone of voice reveal displeasure in current company. It's left for the signal's receiver to judge the reliability of a signal. Though many of these signals are given without thought, they serve as efficient cues in groups.

Groups use signals to coordinate cooperative action. Organized groups, such as a baseball team or military unit, might actively establish gestures and most familiar groups naturally learn to recognize cues to anticipate the needs of others. Coordinating groups that require complicated orchestration often necessitates complex but intuitive non-verbal communication [6].

Conversation Votes, leverages the idea of visual cues in the interface. Cues remain interpretable and subtle, as is body language, while giving participants explicit opportunity to signal. With Conversation Votes we are not trying to eliminate or replace the face-to-face element, but to provide that extra back-channel in face-to-face interaction.

\subsection{Group Awareness}

Shared group displays can alter small group conversations. Simply, labeling individuals as over-participating, participating, and under-participating, participants seek a conversation balanced in contribution [19]. In follow-up work, a suite of conversation visualizations allowed participants to gain insight into their interaction after the session concluded [20]. Post meeting displays, designed to include the conversation history, were found to be more informative for the group as they included a detailed depiction of the verbal exchange.

Collocated and remote groups benefit from cues through heightened group awareness. In collocated programming environments, shared displays highlight changes and conflict in a development group [21]. This visual feedback allows the collocated groups to be more agile in their process: developers saw when they were both editing codependent files and could coordinate their changes more appropriately. In a remote setting, interfaces re-establish status cues that enable effortless coordination when face-to-face. Status cues have been used to indicate a remote presence, the desire to speak in a teleconference, or to indicate confusion in a meeting [22][23].

When introducing anonymity into group awareness, the issue of trusting the signal becomes more important. With fewer repercussions for creating animosity, an anonymous signal has been used to create discord for no reason other than to incite an argument [24]. In Conversation Votes we rely on collocation and mutual accountability to keep the anonymous signals meaningful. People are more trusting and cooperative with known individuals [25][26], and the cues gathered from the visualization are evaluated with knowledge of the all other personal cues in the space.

We explore group awareness in a collocated setting with a mixture of explicit feedback and anonymous cues. Conversation Votes simultaneously presents the aural interaction history annotated with perception cues. 


\subsection{Aural Augmentation}

Sound and speech are experienced serially, one word after another, hindering the ability to review recorded sound more quickly. Searching through archived recordings to find a specific segment of desired information can require listening to the full recording. In research, some provide tags to index important moments for later review, a method similar to random access [27]. Others have hastened aural review by speeding up the playback speed, removing silence, and providing automatically generated transcripts [28][29][30].

Rather than focusing entirely on word content, others provide a visual display to summarize the aural contribution. Visiphone displayed a synchronous continuous depiction activity in each remote space by monitoring the volume at each end and combining them into a common visualization [8]. As conversational dominance was one of the most cited observations in Visiphone, it was recommended for use in marriage counseling sessions as a demonstration tool. Another project, the Conversation Clock, provided a persistent history of conversation [31]. The Conversation Clock produced augmented visual cues of conversation domination, interruption, turn taking, mimicry, and more based solely on the aural input of participants. Using this visualization, participants reported increased awareness of conversational patterns and found the visualized history revealed patterns that were otherwise undetected.

Audio augmentation and visualization has been explored in artistic works focused on collocated audio and the interaction of sound. These works use aural input to create continually changing visualizations. For example, Levin's work In-situ detects phonemes and produces depictions of each based on the aural characteristics of any utterance [32].

These works demonstrate the benefit visualization provides in understanding audio and interaction. Conversation Votes cues act as powerful tags to visually annotate audio during conversation. While we do not include text, transcripts, and speech recognition in our visualizations, the annotated visualization salient moments of conversation are highlighted through participant feedback.

\section{Conversation Votes}

Conversation Votes falls in a category of visualization called social mirrors [31]. By capturing and visualizing social activity back into a group. In the visualization, a shared image of the participants' changing interaction allows direct comparison between oneself and all others at the table.

We designed the anonymous back-channel as a medium for those with less social standing to offer their opinions [10]. In large groups, conference calls, or public meetings there is not always the opportunity for all to speak; participation favors those with higher social rank. The Conversation Votes visualization annotates aural activity with explicit voting feedback on the tabletop display. The resulting public image conveys the group's opinion of the conversation and denotes salient moments. Additionally, the anonymous votes further lower the social cost of entry into conversation allowing a wider swath of views to be discussed [33]. 
Conversation Votes extends the idea of the social mirror described in [31]. As a social mirror it provides a real-time common visualization for a group of four participants in a conversation. The visualization presents a structured timeline that highlights speech and voting activity integrated into the physical environment. Individuals can access additional knowledge and the cues in a small group without drawing visual attention away from the group.

\subsection{The Timeline and History}

In designing a table visualization integrated into conversation, we aim to convey the most important information at a glance. Our structured timeline highlights the most recent past while summarizing interaction history.

The Conversation Votes visualization presents the passage of time as a sequence of rectangular bars. The length of each rectangle represents the average audio sample for a single second. As shown in Figure 2a, rectangular samples progress through the table's center. This center progression shows one minute of elapsed conversation. Its central location on the table provides a detailed view of the interaction most recent in history.

Either side of the center progression depicts older minutes in the conversation (Figure 1b). Details are smaller and less visible; participants to get a higher-level view of who talks, how long people talk, and who received votes. The history stacks accumulate to 16 minutes, each stack showing one minute of samples. The leftmost stack of rectangles in our images indicates the most current minute. The full history is replicated above and below the central progression, making it easily visible from any seat at the table. As each minute ends, all completed minutes slide over to make room for a new minute (Figure $2 \mathrm{~b}$ ). The timeline and history provide the structure to view the individual contributions that make up conversational cues.
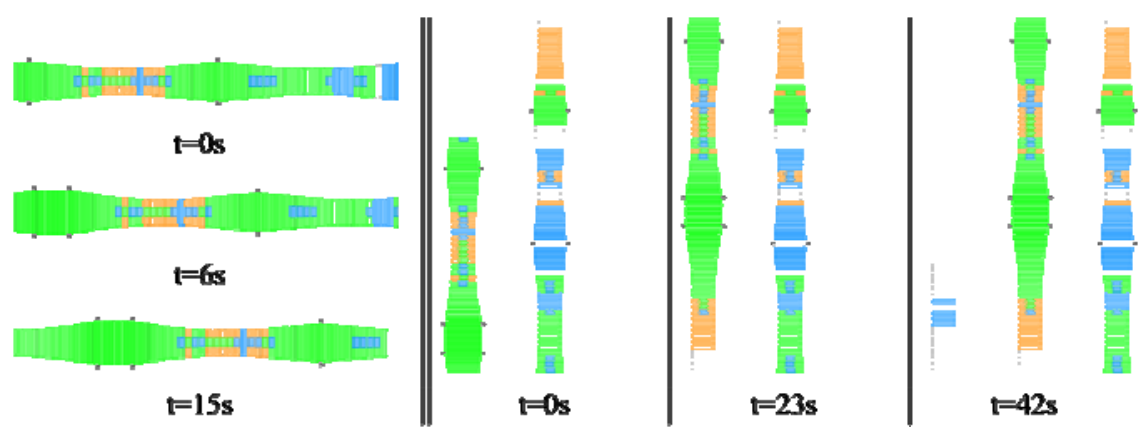

Fig. 2. Figure 2a shows the progression of the central history. Each second, a new sample is taken and appended to the leftmost end of the progression. All bars in the progression slide to the right to make room. Dotted rectangles mark the time a vote took place and simultaneous speech can be seen as multicolored bars. Figure $2 \mathrm{~b}$ demonstrates how the historical stacks move over time. Each line of samples represents a single minute of time, the most recent picture furthest to the left. A line fills over the span of a minute and moves to the right to make room for the next.' 


\subsection{Contribution and Voting}

Conversation Votes shows interaction by noting who spoke when and for how long. Individual microphones monitor each speaker, and unique colors identify each microphone in the visualization. With no votes, the visualization provides a simple colorcoded view of who spoke in the conversation. Initially each sample is of uniform length, favoring no individual.

While the visualization automatically captures a conversation's aural features, it relies on the participants' votes to highlight a salient moment and provide feedback. Participants cast positive votes and negative votes (Figure 3) at any time during the conversation to indicate approval or disapproval respectively. A positive vote increases the size of the sampled bars while a negative vote has the opposite effect on the same set of samples. Additionally, a positive vote brightens the colored interior of the bar while a negative vote causes the color to fade into the background. The vote influences adjacent bars for visual impact while acknowledging the difficulty in pressing a button at the exact moment an utterance occurs. While a vote will always occur after the exact instance that inspired a participant to vote, adjacent bars are adjusted in both directions under the assumption that salient moments of conversation might continue after the button press.

Voting buttons can be held in one's hand discreetly and pressed with little effort. By using his or her two buttons, each listener alters the representation of the current speakers. A viewer sees which speakers provided a greater positive contribution to conversation by examining the full history. Larger and more saturated bars distinguish positive contribution at a glance. In our pilot study, participants found negative votes hurtful. Some voiced their concern about the animosity created. No one wanted to end his or her utterance on a negative note when in such a small group. These same participants agreed that it might be much more useful in larger, room-filling, groups and crowds. For this reason, we removed the negative voting button for our full study of small groups.
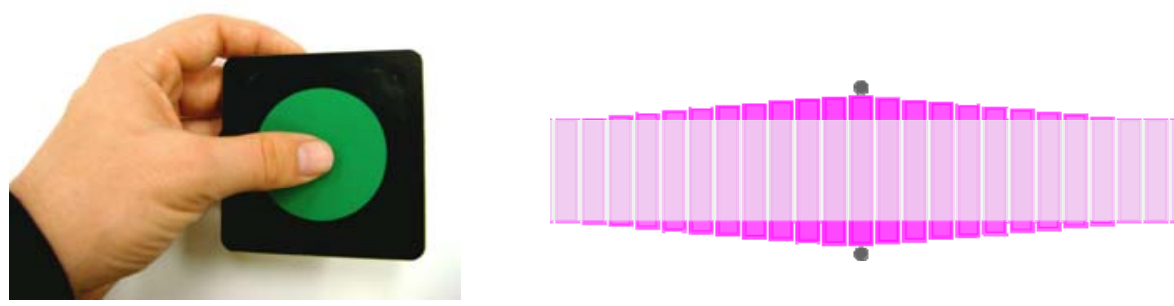

Fig. 3. The voting button (left) is a handheld plate with a colored circle indicating where to push. The button could be held discreetly under the table. There was no tactile feedback in this button, a light press was sufficient to vote and alter the visualization. The effect of a positive (right) lengthens the otherwise uniform bars.

\subsection{Simultaneous Speakers}

Moments of simultaneous vocalization, indicating excitement, agreement, or contention, proved to be among the most salient aural features portrayed by the Conversation 
Clock [31]. We incorporated this visual cue into Conversation Votes by showing the two loudest active speakers in each sample bar. The speaker with greater volume determines the color of the outer bar while embedded rectangle represents the second speaker. Previously, overlapped bars would indicate relative amplitude of each speaker. As the bar length is no longer indicative of amplitude, the decision to only show two speakers makes the visualization more legible.

\section{User Study}

We set out to evaluate the following questions:

Q1: Do less talkative members provide more anonymous feedback?

Q2: Does receiving votes change a person's interactions?

Q3: Will positive feedback disrupt the trend to balance conversation?

Q4: Does voting convey participants' views in the conversation?

\subsection{Method}

We gathered 24 volunteers (13 male / 11 female) to meet in 6 groups. Participants consisted predominately of undergrad and graduate students in engineering disciplines. We asked each group to meet once in the our lab and take part in three debates.

The four participants of a group sat at our rectangular table, two per side on the long side. Prior to beginning the study, lapel microphones were clipped to each participant's collar before calibrating the microphone sensitivity. Participants were also given a single button and told to indicate their approval and encouragement of the current speaker by pressing it. As there is little sensory feedback when pressing the buttons, all participants first tested a button press before beginning the session.

A full session lasted about 1.5 hours and consisted of three 15-minute mini-session debates. In order to provide a base level of activity for comparison, the first mini sessions were conducted without the visualization projected. In this session, participants were not aware of the visualization's appearance and voted knowing only they were marking positive moments in conversation. The second mini-session began with a demonstration of the previous topic's visualization, an explanation of how conversation was depicted, and a live demonstration. The visualization was projected onto the table for the duration of the topic (Figure 1a). The final mini-session was without the visualization to offer a comparison with the baseline.

Topics for each mini-session were chosen from a collection of debate topics for youth debate groups that would be familiar and easily understood by most participants: the minimum age to allow voting, the establishment of national ID cards, and banning smoking in public places. The debate nature of the discussion was to provoke a confrontational style of conversation. Debate questions were assigned prior to each mini-session, and each group received a unique ordering. Participants were free to argue either side of the issue, explore a topic, and switch sides during discussion.

For each mini-session, we logged the aural activity and button presses used to generate the Conversation Votes visualization. For each individual we monitored their participation and noted how often they Lead conversation, how many Turns and the Turn Length, and the number of Votes. These measurements are all straightforward 
and simply logged during conversation. We also generated a measure of Voting Effect to represent the visual "bump" a participant receives (Figure 3). Voting effect can be calculated as the increase of a participant's graphical rendering from a baseline of no votes. All of these measures were normalized to be per minute for comparison across groups.

To measure individual perspective on the conversation, mini-sessions concluded with the same brief questionnaire. We presented participants with three, seven point, Likert Scales to measure how adequately everyone's viewpoints were Represented, how Comfortable participants were in the discussion, and how much their opinion was Altered due the discussion. Additionally, we asked all participants to notate the degree of contribution each group member made to the conversation.

The second session questionnaire included an additional set of seven point Likert scales to investigate the visualization awareness, visualization accuracy, level of voting anonymity, and degree of altered participation due to the visualization.

As part of the survey following each mini-session, participants were asked to estimate the total contribution during conversation. We compared this attribution to the logged lead data to calculate the Estimation Error in all conditions.

\subsection{Results}

Overall, our investigation demonstrates that anonymous voting creates an effective back-channel to enable some, though not all, to better assert themselves in conversation. Though this group was not the less talkative members that we expected, those enabled by the back-channel felt the un-augmented conversation was less inclusive of all viewpoints.

To investigate our hypotheses, participants were classified and divided for comparative analysis. Similar to prior work, groups are divided based on aural participation in the initial session; Heavy contributors spoke more than the leads per minute median value while light contributors spoke below that same threshold. To explore voting, participants were grouped into active voters and less active voters to examine how the voted and divided into heavily supported and lightly supported based on the visual effect of received votes.

Table 1. The table below summarizes the second session's likert scale survey. Participants reported being somewhat altered in their interaction and noticing alteration in others.

(a) Changes over Sessions

\begin{tabular}{rll} 
Measure & F value & p value \\
\hline Leads & $F_{(2,21.3)}=0.25$ & $\boldsymbol{p}<0.79$ \\
Turns & $F_{(2,20.3)}=1.00$ & $\boldsymbol{p}<0.39$ \\
Turn Length & $F_{(2,24.1)}=3.68$ & $\mathbf{p}<\mathbf{0 . 0 4}$ \\
Votes & $F_{(2,19.1)}=0.65$ & $\boldsymbol{p}<0.54$ \\
V. Effect & $F_{(2,38.0)}=1.04$ & $p<0.37$ \\
Represented & $F_{(2,22.6)}=\mathbf{2 . 1 3}$ & $\boldsymbol{p}<0.14$ \\
Comfortable & $F_{(2,22.6)}=0.55$ & $p<0.59$ \\
Opinion & $F_{(2,22.5)}=0.25$ & $p<0.79$ \\
Eirror Est & $F_{(2,24.9)}=3.10$ & $p<0.063$ \\
\hline
\end{tabular}

(b) Likert Results

\begin{tabular}{rc} 
Measure & Result \\
\hline Altered You & 3.38 \\
Attered Others & 3.38 \\
Anomymous & 5.08 \\
Comfortable Voting & 4.33 \\
Look at Vis & 5.16 \\
Encouraged to Speak* & 4.00 \\
ranged from 1-Less to & \\
r-Mone
\end{tabular}


For the statistical analysis, we fit our data to a Linear Mixed Effects Model using Hierarchical Linear Modeling with a repeated condition. A generalized linear model, it is commonly used to address hierarchical data models in social and behavioral sciences when analyzing groups with set hierarchies. Modeling our data as individuals who are a part of a group, we acknowledge individuals are not independent observations and account for the variance that naturally occurs between groups. To investigate Q1-Q4 posed earlier, the model also included variables indicating splits defined above.

Table 2. Splitting the participant groups on three different variables, the linear mixed effects model highlights the differing interaction emerging from our split categorizations over time and across the visualization conditions

\begin{tabular}{|c|c|c|c|c|c|c|c|}
\hline & $d f$ & \multicolumn{2}{|c|}{ Participation Split } & \multicolumn{2}{|c|}{ Voting Split } & \multicolumn{2}{|c|}{ Supported Sphit } \\
\hline Lears & $(2,21.3)$ & $F=5.42$ & $p<0.012$ & $F=5.35$ & $p<0.013$ & $F=2.85$ & $p<0.080$ \\
\hline Tums & $(2,20.3)$ & $F^{r}=\mathbf{2 . 3 2}$ & $p<0.13$ & $F=6.54$ & $p<0.006$ & $F=0.42$ & $p<0.67$ \\
\hline Turn Length & $(2,24.1)$ & $F=0.21$ & $p<0.82$ & $F=0.55$ & $p<0.59$ & $F=1.08$ & $p<0.36$ \\
\hline Votes & $(2,19.1)$ & $F=1.08$ & $p<0.36$ & $F=1.12$ & $p<0.3$ & $F=2.79$ & 0.087 \\
\hline V. Effect & $(2,38.0)$ & $F=0.30$ & $p<0.75$ & $F=0.48$ & $p<0.65$ & $F=0.03$ & 1.98 \\
\hline Represented & $(2,20.6)$ & $F=0.14$ & $p<0.88$ & $F=4.45$ & $p<0.021$ & $F=1.02$ & $p<0.38$ \\
\hline Comfortable & $(2,22.6)$ & $F^{r}=0.64$ & $p<0.54$ & $F=0.05$ & $p<0.96$ & $F=0.50$ & $p<0.57$ \\
\hline Opinion & $(2,22.5)$ & $F=0.91$ & $p<0.42$ & $F=0.25$ & $p<0.79$ & $F=0.63$ & $p<0.54$ \\
\hline Error Est & $(2,24.9)$ & $F=0.29$ & $p<0.80$ & $F=0.04$ & $p<0.96$ & $F=0.65$ & $p<0.54$ \\
\hline
\end{tabular}

Changes ower Sessions, by sub-categorization

As can be seen in Table 1a, Conversation Votes only altered turn length consistently across all individuals $\left(\mathrm{F}_{(2,24.1)}=3.68, \mathrm{p}<0.04\right)$. Follow-up investigation reveals that turn lengths decreased in the final mini-session. Table 1a also shows error estimation approached significance, indicating that people are modestly better at estimating contribution when they have a visual representation available. Surveys presented during the visualization session indicated participants were aware of stronger changes than these initial results reveal. They noted that both their own and others participation were altered from the previous session (Table 1b). This does turn out to be the case, but it is only after analyzing changes throughout the sessions when split that it becomes apparent.

Few differences could be found, outside of the defining characteristic, by making comparisons between the splits overall. Heavy participants tended to speak about 9 seconds more per minute than the less active participants $\left(\mathrm{F}_{(1,25.1)}=38.83, \mathrm{p}<0.001\right)$ and take an additional turn every two minutes $\left(\mathrm{F}_{(1,21.7)}=19.83, \mathrm{p}<0.001\right)$. Active voters pressed their buttons an additional time every 2 minutes $\left(\mathrm{F}_{(1,23: 0)}=13.47, \mathrm{p}<\right.$ 0.001 ), and heavily supported participants received $10 \%$ more increase in visual prominence than did the remaining participants $\left(\mathrm{F}_{(1,28.2)}=11.69, \mathrm{p}<0.002\right)$. However, we see many more interesting differences when the splits are examined over the course of the sessions (Table 2).

The first split, between heavy and light participants, is motivated by previous work demonstrating visual feedback of group activity tends to balance contribution [19][31]. Our results with the Conversation Votes supports that finding $\left(\mathrm{F}_{(2,21.3)}=\right.$ 5.42, $\mathrm{p}<0.012$ ). Participants noted a change in their debate, stating, "[it] more evenly dispersed conversation. I was less likely to interrupt." Overall, the visualization 


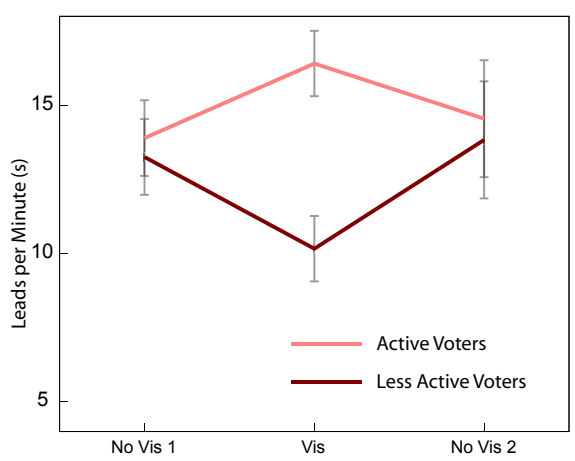

(a) Differences in voter speech

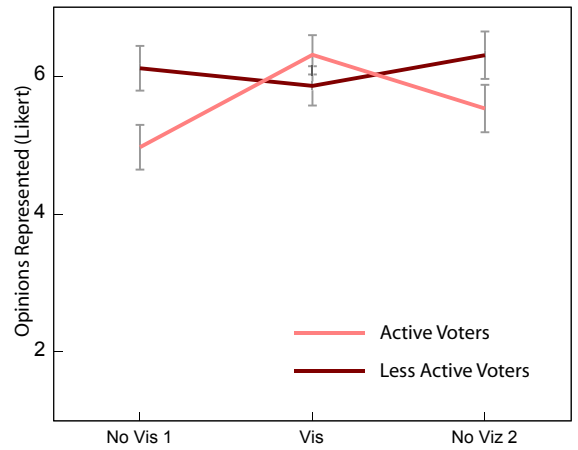

(b) Differences in voter opinion

Fig. 4. Though similar contributions were seen without the visualization, Figure 4a shows active voters increased their contribution while less active voters significantly decreased during the visualization condition. Figure $4 \mathrm{~b}$ shows that the most active voters did not feel representation was comparable until the visualization was present in mini-session two.

encourages a more equitable distribution of contribution and can be considered a balancing element in conversation.

An even more interesting story is told when examining differences between active and less active voters (Table 2). Unlike the balancing seen in the previous split, when active voters can see the results of their votes they drastically increase their lead in the conversation, diverging from the non-voters $\left(\mathrm{F}_{(2,21.3)}=5.35, \mathrm{p}<0.013\right)$. Looking at Figure $4 \mathrm{a}$, we see that both groups are essentially equals in leading conversation with no visualization present, but active voters speak about $30 \%$ more than less active voters with the visualization.

These same active voters reported better representation of opinions when the visualization was shown $\left(\mathrm{F}_{(2,22.6)}=4.45, \mathrm{p}<0.021\right)$. Seen in Figure $4 \mathrm{~b}$, active participants were significantly less satisfied than their less active counterparts that conversation was providing a full representation of viewpoints. Feedback also indicated the backchannel could have been better utilized with a larger vocabulary of signals. One participant stated a negative vote would be particularly useful because he looked when he wanted to move on and "felt someone was talking too much." Though the channel was limited, the visualization allowed this group of active voters not only to speak more, but also to feel more satisfied with the group discussion.

Surprisingly, though voting enables activity and makes participants feel better about the debate, receiving a vote made little difference in our quantitative results. However, it inspired the most conversation amongst the participants.

"You could see when the others agreed with you, so it encouraged you to continue talking."

"I could get a visual grasp of argument/conversation successes (i.e. winning others over)."

"[I would] check if others were agreeing with the point presented (not necessarily by me)." 
In spite of our participants' receptive comments, the last split examining the heavily and lightly supported shows no significant differences, only two notable differences. These notable differences in leads $\left(\mathrm{F}_{(2,21.3)}=2.85, \mathrm{p}<0.080\right)$ and votes $\left(\mathrm{F}_{(2,19.1)}\right.$ $=2.79, \mathrm{p}<0.087)$ fall in line with the above quotes. However, a larger testing pool is needed to confirm those receiving votes become more talkative and more apt to vote during the Conversation Votes sessions.

\section{Discussion}

The results demonstrate that conversation visualization with a voting back-channel can influence conversation and perception. Below is a brief summary of highlights from the qualitative and quantitative highlights, noting how it relates to our original four questions.

Opening a back-channel: With Q1, we sought to show an anonymous back-channel offered an outlet for those reluctant to speak up. We had expected the light contributors would utilize the back-channel while heavy contributors favored speaking. However, our results do not show a significant difference in the voting patterns of heavy and light contributors.

Instead, active and unsatisfied voters increased their participation over the less active voters with the visual cues (Figure 4a) to ensure more opinions were represented (Figure 4b). Qualitative feedback also indicates the back-channel helped the group to better craft their arguments by understanding the group's overall sentiment.

The back-channel did not serve the purpose we had targeted, but it did create a new medium to better shape conversation contribution.

Participants strive for balance: The social mirror encourages people to have a balanced conversation as posed in Q3. Previous work found significant change towards balance when conversation is visualized. Presenting perceived contribution as opposed to raw data did not change this effect.

However, we cannot claim that a balanced conversation is a necessary goal of good conversation. A balanced conversation might be a result of silencing a more informed or provocative speaker. Further study is necessary to investigate the definition of quality in conversation, though our results for Q1 and opening a back-channel indicate that the balance did not lessen the quality of group conversation.

Awareness of Self and Others: With the visualization, participants reported being significantly more aware of others' contributions. They reported checking the visualization for agreements and approval of points. The visualization became a testing ground for ideas and feedback into one's success within the rest of the group. Participants also reported checking for reactions in response to other people's points. For $\mathrm{Q} 4$, we argue the visualization does adequately convey how participation view conversation.

The Voice of the Voter: The heaviest voters were less satisfied with the overall representation of ideas. Their voting could indicate pressure toward other topics. As one participant stated, the vocabulary of feedback needs to be expanded. While we removed negative votes for our study, our quantitative and qualitative results support an 
expanded set of cues. We have shown the visualization provides the necessary backchannel to send cues anonymously, however, the voters desired a back-channel with more than just the positive vote.

Social Mirror Karma: In examining Q2, we cannot definitively say receiving votes changed a person's interaction; our numbers are not strong enough to be certain. However, combined with the qualitative feedback, we hypothesize what further work might show.

Heavily supported individuals can be shown to be more talkative and more active in voting when visual feedback is present. Participating in the conversation and being active in the social mirror seem to correlate with receiving more votes from the remaining participants, in a sense one must give in order to receive. While a participant mentioned the possibility of gaming the system by anonymously voting for himself, an examination of the logs does indicate it occurred in our study.

From our own observation we posit both receiving and casting votes are influenced by engagement in the conversation. A participant in conversation is likely to speak more and vote more when a topic is close to his or her knowledge or interest, encouraging them to influence the social mirror to support their ideas. Future work should consider measuring prior knowledge and taking it into account.

\section{Conclusion}

Conversation is about more than relaying words. Rather than focusing on recreating face-to-face cues in a remote space, our work encourages the exploration of beneficial augmentations for collocated spaces.

We have shown that anonymous back-channels are used to better understand a group and balance participation while conversing. Participants responded in debates based on the feedback they received from the table and reported a heightened awareness of others opinions and their own interaction. Further, we have shown that the heaviest voting block are those who feel conversation is not adequately representing all viewpoints. Though our feedback is limited in vocabulary, Conversation Votes is our first step in enabling the underrepresented voices to be heard in a collocated setting.

\section{References}

1. Duncan, S.: Some signals and rules for taking speaking turns in conversation. Journ of Personality and Social Psyc. (1972)

2. Goffman, E.: The Presentation of Self in Everyday Life. Doubleday Anchor Books (1959)

3. Goffman, E.: On Face-Work. In: Interaction Ritual. Anchor Books (1963)

4. Levinson, S.C.: Pragmatics. Cambridge University Press, Cambridge (1983)

5. Mehrabian, A.: Silent Messages. Wadsworth (1971)

6. Heath, C., Luff, P.: Collaboration and control: Crisis management and multimedia technology in London underground line control rooms. Journal of Computer Supported Cooperative Work 1(1), 24-28 (1992) 
7. Bly, S.A., Harrison, S.R., Irwin, S.: Media spaces: bringing people together in a video, audio, and computing environment. Comm. of ACM (1993)

8. Donath, J., Karahalios, K., Viegas, F.: Visiphone. In: ICAD 2000: International Conference on Auditory Display (2000)

9. Hollan, J., Stornetta, S.: Beyond being there. In: Proceedings of CHI (1992)

10. Sproull, L., Kiesler, S.: Connections: New Ways of Working in the Networked Organization. MIT Press, Cambridge (1991)

11. Saville-Troike, M.: The Ethnography of Communication. Basil Blackwell, Inc., New York (1989)

12. Jones, M.G., Gerig, T.M.: Silent Sixth-Grade Students: Characteristics, Achievement, and Teacher Expectations. The Elementary School Journal 95(2), 169-182 (1994)

13. Baltes, B.B., Dickson, M.W., Sherman, M.P., Bauer, C.C., LaGanke, J.S.: ComputerMediated Communication and Group Decision Making: A Meta-Analysis. Organizational Behavior and Human Decision Processes 87(1), 156-179 (2002)

14. Markus, M.L.: Finding a happy medium: explaining the negative effects of electronic communication on social life at work. ACM Trans. Inf. Syst. 12(2), 119-149 (1994)

15. Tannen, D.: Teachers' Classroom Strategies Should Recognize That Men and Women Use Language Differently. The Chronicle of Higher Education (1991)

16. Tannen, D.: Gender and Discourse. Oxford University Press, Oxford (1994)

17. Chaika, E.: Language: The Social Mirror. Heinle and Heinle Publishers (1994)

18. Hauser, M.D., Chomsky, N., Fitch, W.T.: The Faculty of Language: What Is It, Who Has It, and How Did It Evolve? Science 298, 1569-1579 (2002)

19. DiMicco, J.M., Pandolfo, A., Bender, W.: Influencing group participation with a shared display. In: Proceedings of CSCW (2004)

20. DiMicco, J.M., Bender, W.: Group Reactions to Visual Feedback Tools. In: Persuasive Technology (2007)

21. Biehl, J.T., Czerwinski, M., Smith, G., Robertson, G.G.: FASTDash: a visual dashboard for fostering awareness in software teams. In: Proceedings of CHI (2007)

22. Ding, X., Erickson, T., Kellogg, W.A., Levy, S., Christensen, J.E., Sussman, J., Wolf, T.V., Bennett, W.E.: An empirical study of the use of visually enhanced voip audio conferencing: the case of IEAC. In: Proceedings of CHI (2007)

23. Yankelovich, N., Walker, W., Roberts, P., Wessler, M., Kaplan, J., Provino, J.: Meeting central: making distributed meetings more effective. In: Proceedings of CSCW (2004)

24. Donath, J.S.: Identity and deception in the virtual community. Communities in cyberspace (1999)

25. Bradner, E., Mark, G.: Why distance matters: effects on cooperation, persuasion and deception. In: Proceedings of CSCW (2002)

26. Rocco, E.: Trust breaks down in electronic contexts but can be repaired by some initial face-to-face contact. In: Proceedings of CHI (1998)

27. Degen, L., Mander, R., Salomon, G.: Working with audio: integrating personal tape recorders and desktop computers. In: Proceedings of CHI, New York, NY, USA (1992)

28. Arons, B.: SpeechSkimmer: interactively skimming recorded speech. In: Proceedings of UIST (1993)

29. Vemuri, S., DeCamp, P., Bender, W., Schmandt, C.: Improving speech playback using time-compression and speech recognition. In: Proceedings of CHI (2004)

30. Vemuri, S., Schmandt, C., Bender, W.: iRemember: a personal, long-term memory prosthesis. In: Proceedings on Continuous archival and retrival of personal experences, pp. 6574. ACM, New York (2006) 
31. Bergstrom, T., Karahalios, K.: Seeing More: Visualizing Audio Cues. In: Proceedings of Interact (2007)

32. Levin, G., Lieberman, Z.: In-situ speech visualization in real-time interactive installation and performance. In: Proceedings of the symposium on Non-photorealistic animation and rendering (2004)

33. Kiesler, S., Siegel, J., Timothy, W.M.: Social psychological aspects of computer-mediated communication, 657-682 (1988) (Reprint) 